\title{
Performance Enhancement for Adaptive Beam-Forming Application Based Hybrid PSOGSA Algorithm
}

\author{
Ahmed Magdy1, Osama M. EL-Ghandour², Hesham F. A. Hamed1 \\ ${ }^{1}$ Department of Electrical Engineering, Minia University, Minia, Egypt \\ ${ }^{2}$ Department of Electronics, Communications and Computer Engineering, Helwan University, Cairo, Egypt \\ Email: eng ahmedmagdy86@yahoo.com
}

Received 16 March 2015; accepted 21 April 2015; published 24 April 2015

Copyright (C) 2015 by authors and Scientific Research Publishing Inc.

This work is licensed under the Creative Commons Attribution International License (CC BY).

http://creativecommons.org/licenses/by/4.0/

(c) (i) Open Access

\begin{abstract}
Recently researchers were interested in hybrid algorithms for optimization problems for several communication systems. In this paper, a novel algorithm based on hybrid PSOGSA technique (combination of Gravitational Search Algorithm and Particle Swarm Optimization) is presented to enhance the performance analysis of beam-forming for smart antennas systems using $\mathrm{N}$ elements for Uniform Circular Array (UCA) geometry. Complex excitations (phases) of the array radiation pattern are optimized using hybrid PSOGSA technique for a set of simultaneously incident signals. Our results have shown tremendous improvement over the previous work was done using Uniform Linear Array (ULA) geometry and standard GSA in terms of normalized array factor and computational speed for normalized fitness values.
\end{abstract}

\section{Keywords}

Smart Antennas, UCA, Optimization Techniques, Beam-Forming

\section{Introduction}

Adaptive beam-forming capabilities for smart antenna arrays are nowadays used in different applications such as suppression and reduction of interference in wireless mobile communication, besides its effects on the overall quality of service [1] [2]. There are different optimization techniques dealing with adaptive beam-forming for smart antennas. Particle Swarm Optimization (PSO), Central Force Optimization (CFO), and Bacterial Swarm Optimization (BSO) are well known global optimization techniques that are based on a nature-inspired heuristic [3]-[5]. It is proven that CFO requires higher computational complexity but on the other hand it has better per- 
formance than PSO in [6].

Recently, Gravitational Search Algorithm (GSA) is considered as a new optimization technique based on the law of gravity and mass interaction [7]. Where a set of various standard benchmark functions, synthesis of thinned scanned concentric ring array antenna and a fully digital controlled reconfigurable concentric ring array antenna problems were examined in [8] [9]. In most cases the GSA provided superior or at least comparable results with PSO and CFO. The GSA was proposed in [10] [11] for calculating the dimensions of a rectangular patch antenna, and for Direction of Arrival (DOA) estimation using a Uniform Circular Array (UCA) of 12 elements based on maximum likelihood (ML) criteria and showed better performance results over PSO and multiple signal classification (MUSIC) in terms of computational time for fitness function and RMSE. In [12], planar ultra-wide band (UWB) antennas with irregular radiator shapes were designed using GSA and compared with those obtained using CFO algorithm, it was found that the GSA gave better performance than CFO. In [13], GSA was implemented using Open-MP and its results outperformed the PSO by $65.09 \%$ in terms of normalized array factor. In [14], an algorithm based on collective animal behaviour (CAB) was used for finding the best optimal non-uniform excitation weights and optimal uniform inter-element spacing for hyper beam-forming of linear antenna arrays. In [15], a new algorithm based on Hybrid Particle Swarm Optimization with Gravitational Search Algorithm (Hybrid PSOGSA) technique was proposed and showed better performance than standard PSO and GSA in terms of computational speed.

In this paper, a novel algorithm that is based on the hybrid PSOGSA technique is developed for optimal beam-forming using ULA and UCA. The goal is to maximize the beam of the radiation pattern towards the intended user or Signal of Interest (SOI) and minimize the beam of the radiation pattern towards Signal Not of Interest (SNOI) based on controlling the complex weights (phase) of ULA or UCA. The paper is organized as follows. In Section 2, the system model and problem formulation for adaptive beam-forming are explained. In Section 3, two models for smart antenna array models are described. However, hybrid PSOGSA algorithm is proposed in Section 4. Simulation results and discussions for beam-forming are discussed in Section 5. Finally, the conclusion is presented in Section 6.

\section{Problem Formulation}

Smart antenna based on UCA topology by using N elements is showed in Figure 1. In this section, 12 elements in array structure are distributed uniformly along the circle of radius $r$, where $\theta$ is the azimuth angle, $\lambda$ is the wavelength, the angle between adjacent elements is $\theta_{0}$, and $d=0.5 \lambda$ is the space between two adjacent elements.

In the synthesis of beam-forming, the complex excitation for each element must be optimized to minimize radiation power intensity at certain directions and maximize the main-lobes to other directions. The following fitness function must be minimized to maximize the total output power toward the desired signal at $\theta_{i}$ and minimize the total output power in the direction of the interfering signals at $\theta_{j}$.

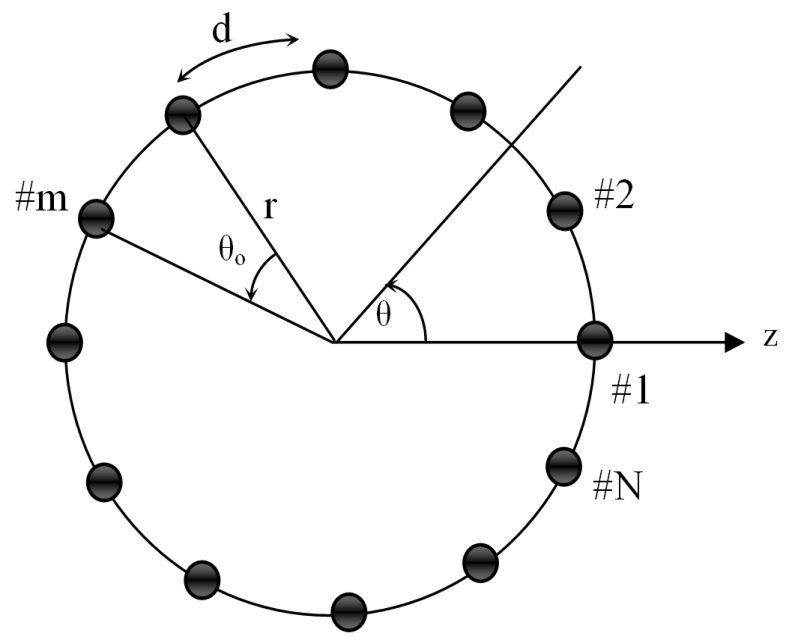

Figure 1. Geometry of the UCA with N elements. 


$$
\text { fitness function }=-\sum_{i=1}^{k} a_{i} \operatorname{AF}\left(\theta_{i}\right)+\sum_{j=1}^{L} b_{j} \operatorname{AF}\left(\theta_{j}\right)
$$

where the number of SOI users is represented in constant $k$, and $L$ represents the number of SNOI hackers. $\operatorname{AF}\left(\theta_{i}\right)$ is the array factor that will be maximized or minimized in specific directions for ULA and UCA using evolutionary algorithms.

$$
\begin{aligned}
& \operatorname{AF}\left(\theta_{i}\right)=\sum_{n=1}^{N} \mathrm{e}^{j\left[\beta * r * \cos \left(\theta-\operatorname{pos}_{n}\right)-\alpha_{n}\right]}, \text { for UCA } \\
& \operatorname{AF}\left(\theta_{i}\right)=\sum_{n=1}^{N} \mathrm{e}^{j\left[\beta *(n-1) * d * \cos (\theta)-\alpha_{n}\right]}, \text { for ULA }
\end{aligned}
$$

where $\alpha_{n}$ represents the complex excitation phase of the $n$-th element, $\operatorname{pos}_{n}$ is the angular position of the $n$-th element, and $\beta$ is the phase shift constant.

\section{Smart Antennas Array Beam-Forming Models}

As two models are proposed as shown in Figure 2, the first model assumes user \#2 as transmitter, desired user \#1 as receiver at desired angle $\mathrm{SOI}=\theta_{d}$ from user \#2, and hacker at angle $\mathrm{SNOI}=\theta_{h}$ from user \#2. In our work, smart antennas array using linear and circular topology are obtained. The second model assumes number of desired users $M$ as SOI angles at $\theta_{i}$.

\section{Hybrid PSOGSA Algorithm Optimization Technique}

Several different hybridization methods for heuristic algorithms was presented in [16], when two different algorithms can be hybridized in high-level or low-level with relay or co-evolutionary method as homogeneous or heterogeneous. In 1995, Kennedy and Eberhart surprised the world by PSO as shown in [3]. PSO was improved by several researchers when many interest variants were developed as shown in [4]. PSO depended on two main equations for updating the velocity and position as follow:

$$
\begin{gathered}
v_{i D}^{k+1}=w v_{i D}^{k}+\text { C1Rand } 1\left(\text { pbest }_{i D}-x_{i D}^{k}\right)+\text { C2Rand } 2\left(\text { gbest }_{D}-x_{i D}^{k}\right) \\
x_{i D}^{k+1}=x_{i D}^{k}+v_{i D}^{k+1}
\end{gathered}
$$

where $x_{i D}\left(x_{i 1}, x_{i 2}, \cdots, x_{i D}\right)$ and $v_{i D}\left(v_{i 1}, v_{i 2}, \cdots, v_{i D}\right)$ are position and variation in position (velocity) of the $i$-th particle for an D-dimensional problem, respectively. pbest ${ }_{i D}\left(\right.$ pbest $_{i 1}$, pbest $_{i 2}, \cdots$, pbest $\left._{i D}\right)$ and gbest $_{D}$ (gbest $_{1}$, gbest $_{2}, \cdots$, gbest ${ }_{D}$ ) represent for personal best position of the $i$-th particle and global best position vector, respectively. C1 and C2 are the acceleration constants, which represent the weighting of stochastic acceleration terms that pull each particle towards pbest and gbest positions. Rand 1 and Rand 2 are two random numbers in the range $[0,1], w$ is the inertia weight introduced to balance between the global and local search abilities

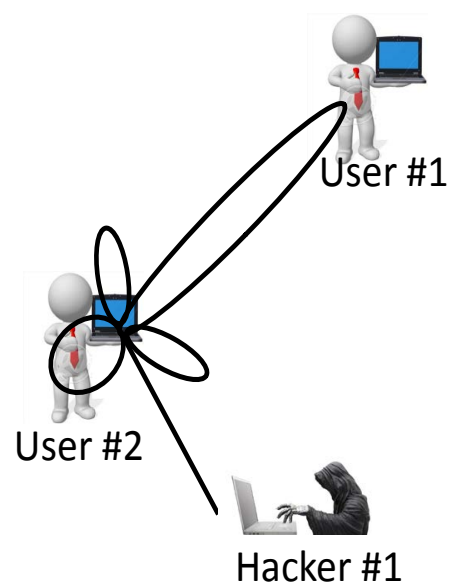

(a)

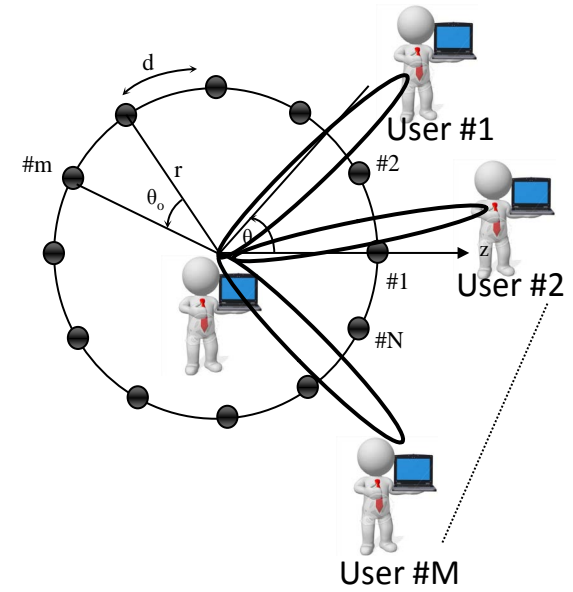

(b)

Figure 2. Two models for smart antennas array. 
in terms of $k$ iterations.

Recently, Gravitational Search Algorithm (GSA) was provided as an optimization problem based on the law of gravity and mass interaction [7]. The algorithm considered agents as objects consisting of different masses. Each agent in GSA was specified by four parameters: position of the mass in $d$-th dimension, inertia mass, active gravitational mass and passive gravitational mass. At each iteration, perform of the fitness evolution for all agents and also compute the best and worst fitness defined as below (for minimization problems):

$$
\begin{aligned}
\operatorname{best}(t) & =\min _{j \in\{1, \cdots, K\}} \mathrm{fit}_{j}(t) \\
\operatorname{worst}(t) & =\max _{j \in\{1, \cdots, K\}} \mathrm{fit}_{j}(t)
\end{aligned}
$$

where fit $_{j}(t)$ represents the fitness of the $j$-th agent at iteration $t$, best $(t)$ and worst $(t)$ represents the best and worst fitness at generation $t$. Then the mass of the agents (gravitational and inertia masses) can be calculated as: $M_{a i}=M_{p i}=M_{i i}=M_{i} ; \quad i=1,2, \cdots, K$

$$
\begin{gathered}
m_{i}(t)=\left[\operatorname{fit}_{i}(t)-\text { worst }_{i}(t)\right] /[\operatorname{best}(t)-\operatorname{worst}(t)] \\
M_{i}(t)=m_{i}(t) / \sum_{j=1}^{K} m_{i}(t)
\end{gathered}
$$

where $M_{a i}$ is the active gravitational mass of the $i$-th agent, and $M_{p i}$ is the passive gravitational mass of the i-th agent.

In this paper, PSO with GSA was hybridized using low-level co-evolutionary heterogeneous hybrid. The hybrid is low-level because the functionality of both algorithms is combined. It is co-evolutionary because both algorithms aren't used one after another but run in parallel. It is heterogeneous because there are two different algorithms that are involved to produce final results. The basic idea of hybrid PSOGSA is to combine the ability of social thinking (gbest) in PSO with the local search capability of GSA. In order to combine these algorithms, updating velocity is proposed as follow:

$$
v_{i}(t+1)=w * v_{i}(t)+\mathrm{C} 1 * \text { Rand } * a_{i}(t)+\mathrm{C} 2 * \text { Rand } *\left(\text { gbest }-x_{i}(t)\right)
$$

where $v_{i}(t)$ is the velocity of agent $i$ at iteration $t, w$, Rand, gbest, C1 and C2 are taken from PSO algorithm. On the other hand, from GSA in [7], $a_{i}(t)$ is the acceleration of agent $i$ at iteration $t$ and can be calculated by

$$
a_{i}^{d}(t)=F_{i}^{d}(t) / M_{i i}(t)
$$

where $F_{i}^{d}(t)$ is the total force acting on $i$-th agent calculated as:

$$
F_{i}^{d}(t)=\sum_{i=1, j \neq 1} \operatorname{rand}_{j} F_{i j}^{d}(t)
$$

where $F_{i j}^{d}(t)$ is the force acting on agent $i$ from agent $j$ at $d$-th dimension and $t$-th iteration and is computed as below:

$$
F_{i j}^{d}(t)=G(t) M_{p i}(t) M_{a j}(t)\left(x_{j}^{d}(t)-x_{i}^{d}(t)\right) /\left[R_{i j}(t)+\epsilon\right]
$$

where $R_{i j}(t)$ is the Euclidian distance between two agents $i$ and $j$ at iteration $t, G(t)$ is the computed gravitational constant at the same iteration, and $\epsilon$ is a small constant.

$$
G(t)=G_{0} \mathrm{e}^{(\alpha t / T)}
$$

In this problem $G_{0}$ is set to $100, \alpha$ is set to 20 and $T$ is the total number of iterations. In each iteration, the positions of particles are updated as follow:

$$
x_{i}(t+1)=x_{i}(t)+v_{i}(t+1)
$$

In hybrid PSOGSA [15], firstly, all agents are randomly initialized. Each agent is considered as a candidate solution. As can be seen in Figure 3, after initialization, evaluate the fitness function based on Equation (1). Gravitational force, gravitational constant, and resultant forces among agents are calculated using Equations (12), (14) and (13) respectively. After that, the accelerations of particles are defined as Equation (11). In each iteration, 


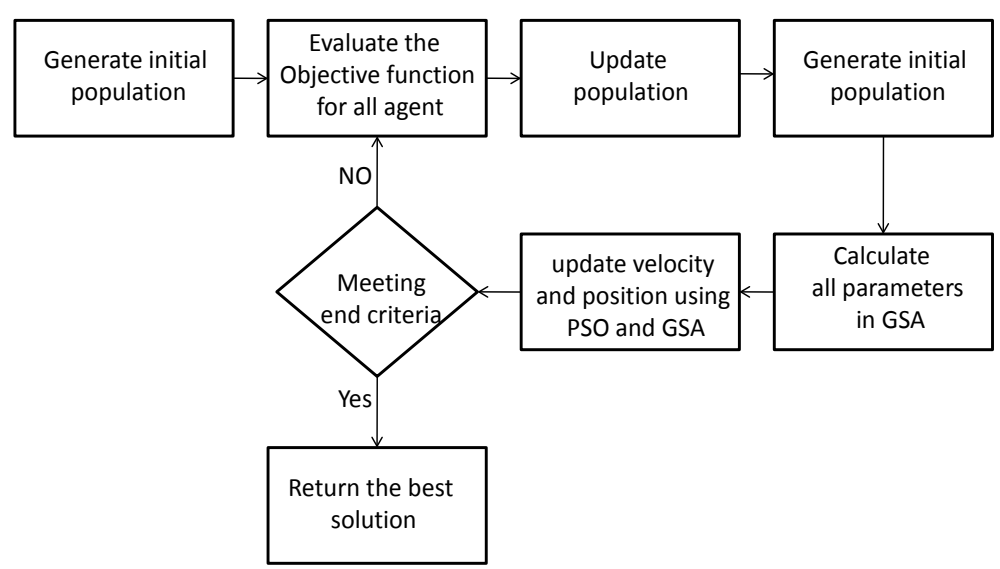

Figure 3. Flow chart for steps in hybrid PSOGSA algorithm.

the best solution so far (gbest) must be updated. After calculating the accelerations and with updating the best solution so far, the velocities and the positions of all agents can be updated using Equations (10) and (15) respectively. Finally, after agents are updated, the process of updating velocities and positions will be stopped by meeting an end criterion.

\section{Simulation and Discussion Results}

To validate the above analysis, we have developed a custom event driven simulator using Matlab package. In this section, the capability of hybrid PSOGSA technique for adaptive beam-forming with a UCA is studied.

In this section two models are discussed, the first model is shown in Section 3. Figures 4-6 show beam patterns in comparison with smart antenna for different geometries (UCA and ULA) from user \#2 to user \#1 (SOI = $\left.30^{\circ}\right)$ and from user $\# 2$ to hacker $\# 1$ (SNOI $=-30^{\circ}\left(330^{\circ}\right)$ or $-20^{\circ}\left(340^{\circ}\right)$ or $-40^{\circ}\left(320^{\circ}\right)$ ). Figure $4(\mathrm{a})$ and Figure 4(b) is obtained for normalized array factor comparison using ULA and UCA by hybrid PSOGSA at SOI $30^{\circ}$ and SNOI $-30^{\circ}\left(330^{\circ}\right)$ in polar and rectangular representation respectively. It is found that only UCA topology has capability to direct the main beam toward user \#1 (SOI) and null at hacker \#1 (SNOI). It is clear that the directed power toward the intended direction $\left(30^{\circ}\right)$ using UCA is better than that obtained by ULA by approximately 55\% as shown in Figure 4(a) (more than $6 \mathrm{~dB}$ as shown in Figure 4(b)). On the other hand, the directed null (zero power) toward the intended direction $\left(-30^{\circ}\right)$ using UCA is better than that obtained by ULA by approximately $35 \mathrm{~dB}$ as shown in Figure 4(b).

Figure 5(a) and Figure 5(b) shows SOI at $30^{\circ}$ and a changed value $-40^{\circ}\left(320^{\circ}\right)$ for SNOI. Accordingly the results showed an improvement of $17 \%$ (more than $1.5 \mathrm{~dB}$ ) and approximately $14 \mathrm{~dB}$ for SOI and SNOI respectively.

Figure 6(a) and Figure 6(b) shows SOI at $30^{\circ}$ and a changed value $-20^{\circ}\left(340^{\circ}\right)$ for SNOI. Accordingly the results showed an improvement of $35 \%$ (more than $3.5 \mathrm{~dB}$ ) and approximately $5 \mathrm{~dB}$ for SOI and SNOI respectively.

Also, it can be noticed that an extra undesired main beam in the broadside direction is obtained in the ULA geometry. Therefore, the first case is the worst case in ULA because SNOI $=-30^{\circ}\left(330^{\circ}\right)=360-\mathrm{SOI}$ (broadside direction). In general, the results obtained by UCA are better than those obtained from ULA which used in [14] for all directions in all scenarios.

The second model is shown in Section 3. Figure 7 shows two scenarios normalized array factor comparison and normalized fitness functions versus iteration number using hybrid PSOGSA and GSA. The hybrid PSOGSA is used to adjust the weights of phase shift of the excitation of each element of the UCA array for beam synthesis to maximize the output power toward the desired signals at $\theta_{i}$ (scenario \#1 two users at: $\theta_{i}=\left[30^{\circ}, 0^{\circ}\right]$, scenario \#2 five users at: $\theta_{i}=\left[30^{\circ}, 0^{\circ},-30^{\circ}, 90^{\circ}, 120^{\circ}\right]$ ). To validate the algorithm, the results are compared to those obtained using GSA which used in [13]. Both algorithms are employed with a population size of 30 and 150 iterations. First part in Figure 7(a) and Figure 7(b) shows the radiation pattern for the case of feeding each element of the UCA with the original precise values of phases optimized by hybrid PSOGSA and GSA for scenarios \#1 and \#2. Part two in Figure 7(a) and Figure 7(b) shows the normalized global best fitness of the hybrid PSOGSA and GSA algorithms for adaptive beam-forming of the array feeding. Comparing the global best 


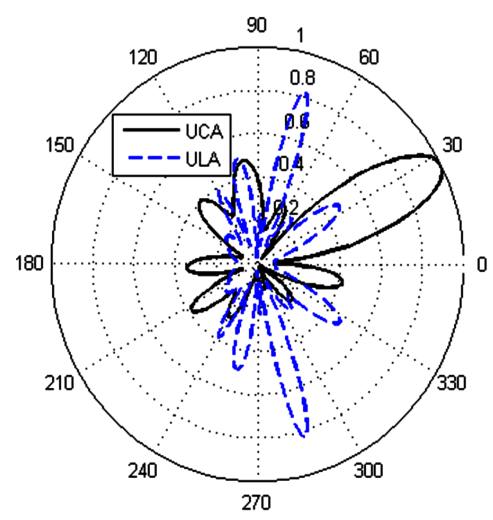

(a)

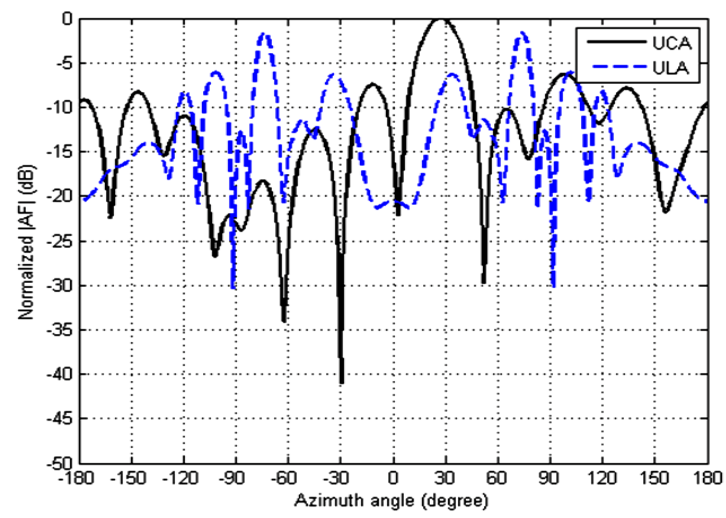

(b)

Figure 4. Normalized array factor comparison using ULA and UCA by hybrid PSOGSA at SOI $30^{\circ}$ and SNOI $-30^{\circ}\left(330^{\circ}\right)$. (a) Polar representation; (b) Rectangular representation.

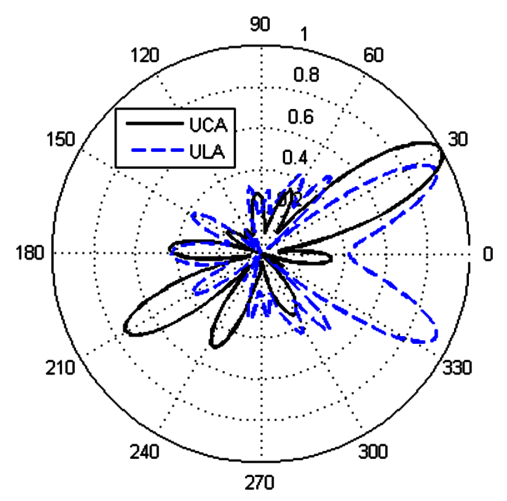

(a)

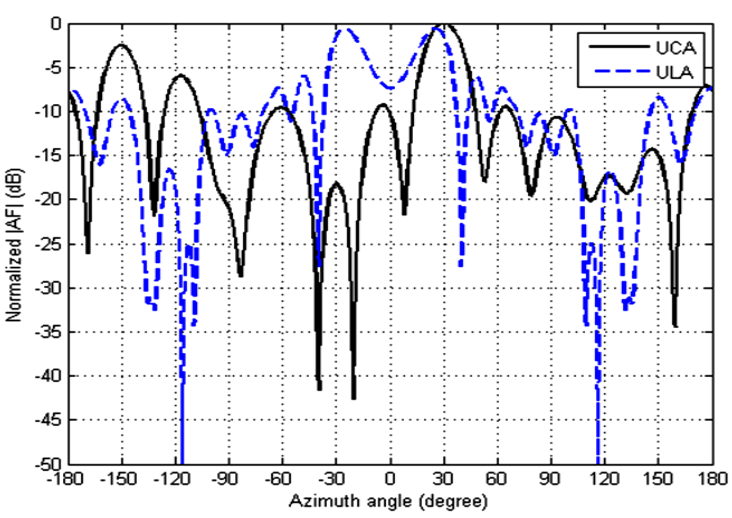

(b)

Figure 5. Normalized array factor comparison using ULA and UCA by hybrid PSOGSA at SOI $30^{\circ}$ and SNOI $-40^{\circ}\left(320^{\circ}\right)$. (a) Polar representation; (b) Rectangular representation.

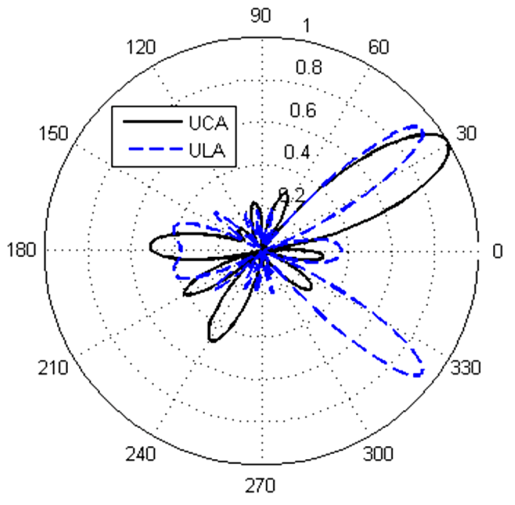

(a)

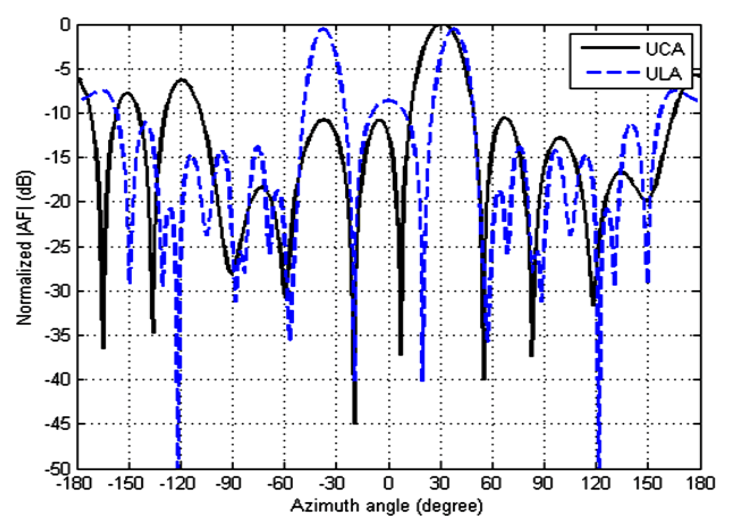

(b)

Figure 6. Normalized array factor comparison using ULA and UCA by hybrid PSOGSA at SOI $30^{\circ}$ and SNOI $-20^{\circ}\left(340^{\circ}\right)$. (a) Polar representation; (b) Rectangular representation.

fitness of the two algorithms, it is found that the hybrid PSOGSA converges faster than GSA with a better performance in terms of computed final fitness values.

The pervious Figure 7 indicates that the hybrid PSOGSA in general outperforms the GSA in precise feedings. Table 1 shows the corresponding normalized $|\mathrm{AF}|$ values at the SOI directions for different scenarios. As shown, 

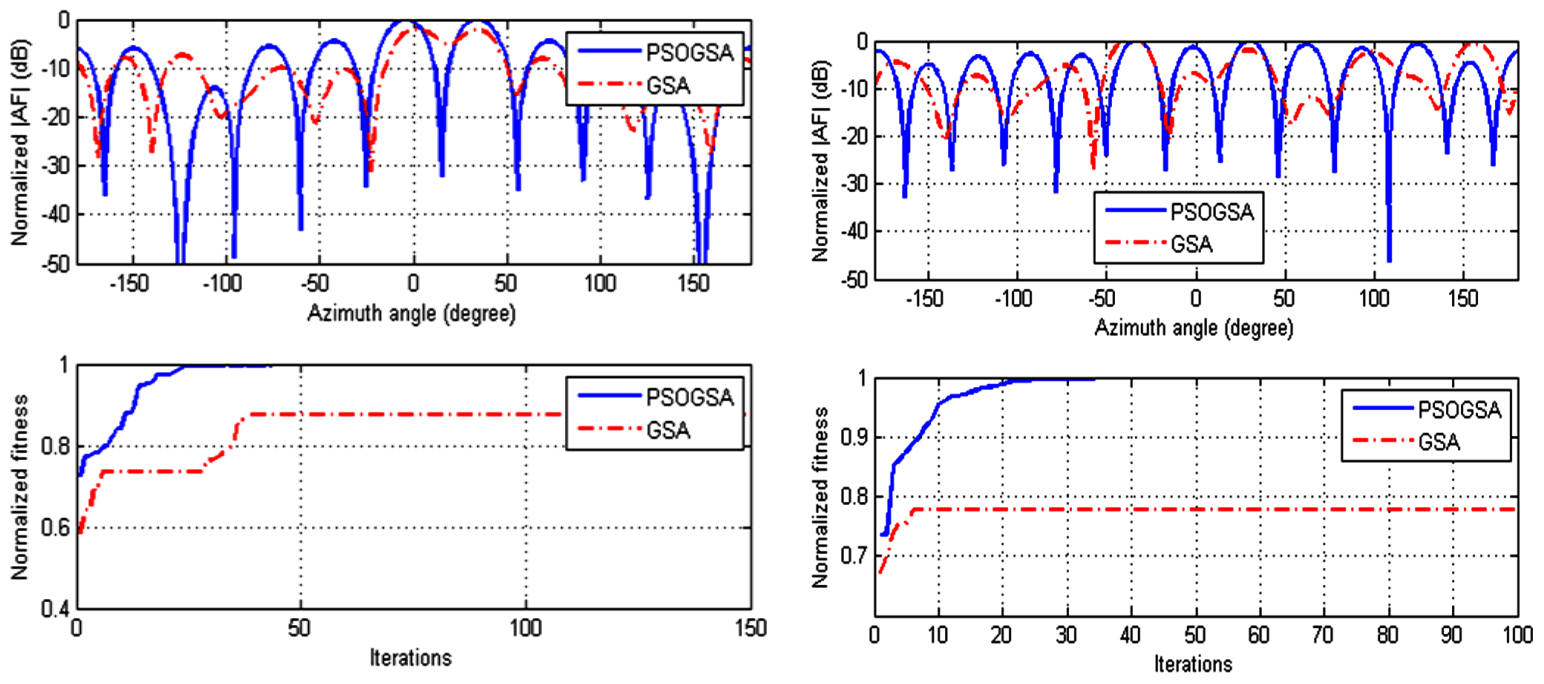

(a)

(b)

Figure 7. Two scenarios normalized array factor comparison and normalized fitness functions versus iteration number using Hybrid PSOGSA and GSA. (a) Scenario \#1; (b) Scenario \#2.

Table 1. The corresponding normalized $|\mathrm{AF}|$ values at the SOI directions for different scenarios.

\begin{tabular}{|c|c|c|c|c|c|c|c|c|c|c|}
\hline & \multirow[b]{2}{*}{ Desired Angles $\theta_{i}$} & \multicolumn{3}{|c|}{ Scenario \#1 } & \multicolumn{6}{|c|}{ Scenario \#2 } \\
\hline & & $0^{\circ}$ & $30^{\circ}$ & $\begin{array}{c}\text { Average } \\
\text { Normalized }|\mathrm{AF}|\end{array}$ & $120^{\circ}$ & $90^{\circ}$ & $30^{\circ}$ & $0^{\circ}$ & $-30^{\circ}$ & $\begin{array}{c}\text { Average Normalized } \\
\qquad|\mathrm{AF}|\end{array}$ \\
\hline \multirow{2}{*}{ 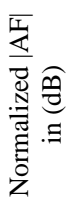 } & Hybrid PSOGSA & 0 & -1 & -0.5 & -1.2 & -1.9 & 0 & -1.53 & -0.6 & -1.046 \\
\hline & Standard GSA & -3.2 & -3.9 & -3.55 & -7.6 & -3.96 & -2 & -6.98 & -0.6 & -4.228 \\
\hline
\end{tabular}

the hybrid PSOGSA is better than GSA by $-3.05 \mathrm{~dB}$ and $-3.182 \mathrm{~dB}$ on the average for scenario \#1 and scenario \#2 respectively.

\section{Conclusion}

In this paper, a new novel (hybrid PSOGSA) technique is proposed with ULA and UCA antenna system for enhancing the performance of adaptive beam-forming in wireless communications applications. The technique is simple and appropriate for real time applications. It is clear that the directed power toward the intended direction (SOI) using UCA is better than that obtained by ULA by approximately 55\% (more than $6 \mathrm{~dB}$ ), $35 \%$ (more than $3.5 \mathrm{~dB}$ ) and $17 \%$ (more than $1.5 \mathrm{~dB}$ ); on the other hand, directed null to SNOI better than ULA by approximately $35 \mathrm{~dB}, 5 \mathrm{~dB}$ and $14 \mathrm{~dB}$ in all cases in the first model in terms of normalized array factor. Simulations of beamforming show accurate results even for a big set of simultaneously incident signals. Strategically pairing GSA with PSO has the desired advantages over GSA. It is found that hybrid PSOGSA is more attractive for beamforming applications and better than GSA with approximately $-3 \mathrm{~dB}$ in average. Via extensive simulation studies, it is demonstrated that hybrid PSOGSA achieves fast and robust global convergence over GSA.

\section{References}

[1] Lehne, P.H. and Pettersen, M. (1999) An Overview of Smart Antenna Technology for Mobile Communications Systems. IEEE Communications Surveys and Tutorials, 2, 2-13.

[2] Chryssomallis, M. (2000) Smart Antennas. IEEE Antennas and Propagation Magazine, 42, 129-136. http://dx.doi.org/10.1109/74.848965 
[3] Kennedy, J. and Eberhart, R. (1995) Particle Swarm Optimization. Proceedings of IEEE International Conference on Neural Networks, Perth, 27 November-01 December 1995, 4, 1942-1948. http://dx.doi.org/10.1109/ICNN.1995.488968

[4] Mahmoud, K.R., Eladawy, M., Bansal, R., Zainud-Deen, S.H. and Ibrahem, S.M.M. (2008) Analysis of Uniform Circular Arrays for Adaptive Beamforming Applications Using Particle Swarm Optimization Algorithm. International Journal of RF and Microwave Computer-Aided Engineering, 18, 42-52. http://dx.doi.org/10.1002/mmce.20265

[5] Formato, R.A. (2007) Central Force Optimization: A New Metaheuristic with Applications in Applied Electromagnetics. Progress in Electromagnetics Research, 77, 425-491. http://dx.doi.org/10.2528/PIER07082403

[6] Mahmoud, K.R. (2011) Central Force Optimization: Nelder-Mead Hybrid Algorithm for Rectangular Microstrip Antenna Design. Electromagnetics, 31, 578-592. http://dx.doi.org/10.1080/02726343.2011.621110

[7] Rashedi, E., Nezamabadi-Pour, H. and Saryazdi, S. (2009) GSA: A Gravitational Search Algorithm. Information Sciences, 179, 2232-2248. http://dx.doi.org/10.1016/j.ins.2009.03.004

[8] Chatterjee, A., Mahanti, G.K. and Pathak, N.N. (2010) Comparative Performance of Gravitational Search Algorithm and Modified Particle Swarm Optimization Algorithm for Synthesis of Thinned Scanned Concentric Ring Array Antenna. Progress in Electromagnetics Research B, 25, 331-348. http://dx.doi.org/10.2528/PIERB10080405

[9] Chatterjee, A., Mahanti, G.K. and Mahapatra, P.R.S. (2011) Design of Fully Digital Controlled Reconfigurable DualBeam Concentric Ring Array Antenna Using Gravitational Search Algorithm. Progress in Electromagnetics Research C, 18, 59-72. http://dx.doi.org/10.2528/PIERC10101806

[10] Altinoz, O.T. and Yilmaz, A.E. (2011) Calculation of Optimized Parameters Of Rectangular Patch Antenna Using Gravitational Search Algorithm. 2011 International Symposium on Innovations in Intelligent Systems and Applications (INISTA), Istanbul, 15-18 June 2011, 349-353. http://dx.doi.org/10.1109/INISTA.2011.5946090

[11] Magdy, A., Mahmoud, K.R., Abdel-Gawad, S.G. and Ibrahim, I.I. (2013) Direction of Arrival Estimation Based on Maximum Likelihood Criteria Using Gravitational Search Algorithm. Progress in Electromagnetics Research Symposium Proceedings, Taipei, 25-28 March 2013, 1162-1167.

[12] Mahmoud, K.R. (2013) UWB Antenna Using Gravitational Search Algorithm. Journal of Engineering Sciences, 41, 1890-1903.

[13] Mahmoud, K.R. and Hamad, S. (2014) Parallel Implementation of Hybrid GSA-NM Algorithm for Adaptive BeamForming Applications. Progress in Electromagnetics Research B, 58, 47-57. http://dx.doi.org/10.2528/PIERB13102106

[14] Ram, G., Mandal, D., Kar, R. and Ghoshal, S.P. (2013) Optimized Hyper Beamforming of Linear Antenna Arrays Using Collective Animal Behaviour. The Scientific World Journal, 2013, Article ID: 982017.

[15] Mirjalili, S. and Hashim, S.Z.M. (2010) A New Hybrid PSOGSA Algorithm for Function Optimization. International Conference on Computer and Information Application (ICCIA), Tianjin, 3-5 December 2010, 374-377.

[16] Talbi, E.G (2002) A Taxonomy of Hybrid Metaheuristic. Journal of Heuristics, 8, 541-546. http://dx.doi.org/10.1023/A:1016540724870 